\title{
Surface layering of liquids: The role of surface tension
}

\author{
Oleg Shpyrko, ${ }^{1}$ Masafumi Fukuto, ${ }^{1}$ Peter Pershan, ${ }^{1}$ Ben Ocko, ${ }^{2}$ Ivan Kuzmenko, ${ }^{3}$ Thomas Gog, ${ }^{3}$ and Moshe Deutsch \\ ${ }^{1}$ Department of Physics, Harvard University, Cambridge, Massachusetts 02138, USA \\ ${ }^{2}$ Department of Physics, Brookhaven National Lab, Upton, New York 11973, USA \\ ${ }^{3}$ CMC-CAT, Argonne National Lab, Argonne, Illinois 60439, USA \\ ${ }^{4}$ Department of Physics, Bar-Ilan University, Ramat-Gan 52900, Israel
}

(Received 30 December 2003; revised manuscript received 25 March 2004; published 30 June 2004)

\begin{abstract}
Recent measurements show that the free surfaces of liquid metals and alloys are always layered, regardless of composition and surface tension; a result supported by three decades of simulations and theory. Recent theoretical work claims, however, that at low enough temperatures the free surfaces of all liquids should become layered, unless preempted by bulk freezing. Using x-ray reflectivity and diffuse scattering measurements we show that there is no observable surface-induced layering in water at $T=298 \mathrm{~K}$, thus highlighting a fundamental difference between dielectric and metallic liquids. The implications of this result for the question in the title are discussed.
\end{abstract}

DOI: 10.1103/PhysRevB.69.245423

PACS number(s): 61.20.Ne, 61.20.Gy

The free surface of liquid metals and alloys were demonstrated experimentally over the last few years to be layered, i.e., to exhibit an atomic-scale oscillatory surface-normal density profile. ${ }^{1-4}$ This is manifested by the appearance of a Bragg-like peak in the x-ray reflectivity (XR) curve, $R\left(q_{z}\right)$, as shown in Fig. 1 for $\mathrm{Ga}^{2}$ The wave-vector-transferposition of the peak, $q_{\text {peak }}$ is related to the layering period $d$ by $q_{\text {peak }}=2 \pi / d$. The layered interface is in a marked contrast with the theoretical description of the liquid-vapor interface of a simple liquid. This theory, prevailing for over a century, depicts the density profile as a monotonic increase from the low density of the vapor and the high density of the bulk liquid. ${ }^{5,6}$ This view was supported by XR measurements on many nonmetallic liquids measured over the last two decades, including water, ${ }^{7}$ alkanes, ${ }^{8}$ and quantum liquids, ${ }^{9}$ which showed no Bragg-like peaks. However, as is discussed below the measurements in all of these studies were restricted to the small $q$ range, i.e., $q_{z} \ll \pi /$ atomic size, and would not have detected surface layering if it existed.

Early simulations on nonmetallic liquids demonstrate that atomic layering is ubiquitous near a hard flat surface and this has been observed for liquid Gallium. ${ }^{10}$ On the other hand, for the liquid-vapor interface it is tempting to think that the large surface tension $\gamma$ of liquid metals such as $\mathrm{Hg}(\gamma \approx 500 \mathrm{mN} / \mathrm{m}), \quad \mathrm{Ga}(\gamma \approx 750 \mathrm{mN} / \mathrm{m})$, and $\mathrm{In}$ $(\gamma \approx 550 \mathrm{mN} / \mathrm{m})$ might be the explanation for the SL observed at their surface. This assumption is partially mitigated by the observation of SL at the free surface of liquid $\mathrm{K}$ where $\gamma \approx 100 \mathrm{mN} / \mathrm{m}$ only. Here we report $\mathrm{x}$-ray scattering results showing that the free surface of water, which has nearly the same surface tension as K, does not exhibit SL features in the reflectivity profiles, thereby suggesting that surface tension by itself does not explain SL. This conclusion rests on the validity of the capillary wave theory (discussed below).

Rice et al. ${ }^{11}$ first predicted SL in liquid metals three decades ago. They argued that the layered interface structure for liquid metals is a consequence of the strong dependence of the effective ion-potential energy on the steeply varying electron density across the liquid/vapor interface. At the lowdensity vapor phase the electrically neutral atoms interact through a van der Waals interaction only. In the metallic, higher density, liquid phase the electrons are delocalized, and the much more complex interactions involve an interplay between a quantum Fermi fluid of free electrons and a classical liquid of charged ion cores. Rice concludes that this substantive change in the effective-ion-potential stabilizes the short-range surface fluctuations with the result that the atoms near the surface form a layered structure. ${ }^{11}$ Calculations employing the glue model of metallic cohesion support these conclusions. ${ }^{12}$ By contrast, Soler et al. ${ }^{13}$ claim that SL is solely due to the formation of a dense layer at the surface. This layer is not restricted to metallic liquids, but may form also in nonmetallic liquids due to nonisotropic interactions, such as remnant covalent bonding in liquid Si (Ref. 13) and the highly directional interactions in liquid crystals, ${ }^{14}$ or in nonuniform Lennard-Jones fluids with unbalanced attractive forces. ${ }^{15}$ Additionally, the SL has been found in colloidal systems,${ }^{16}$ which suggests that layering phenomenon may be

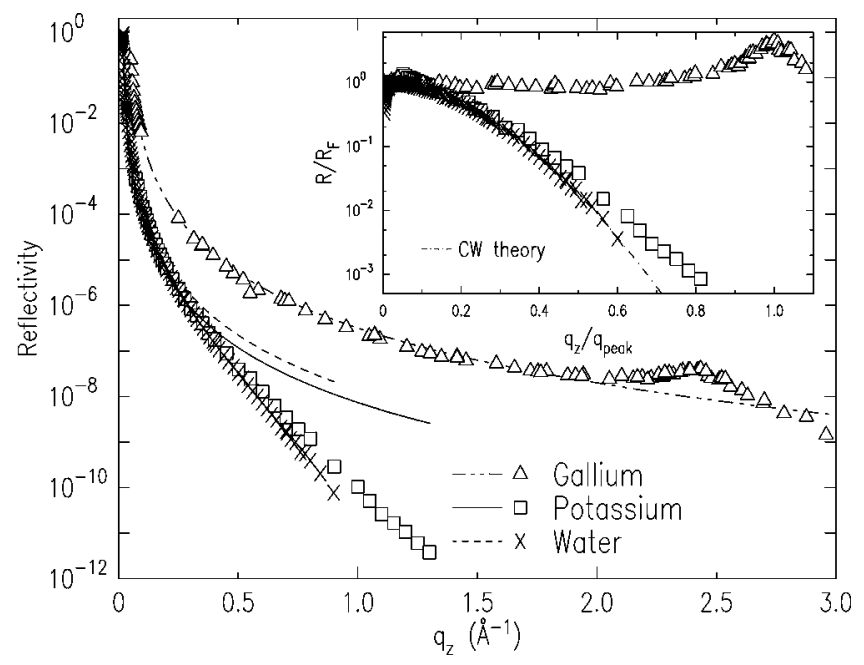

FIG. 1. X-ray reflectivity from free liquid surfaces of the indicated samples. Points, measured $\left[R\left(q_{z}\right)\right]$, lines, calculated for an ideally flat and steplike interface $\left[R_{F}\left(q_{z}\right)\right]$. The inset shows the ratio of the two. 
more fundamental than previously thought. More recently, based on extensive simulations, Chacón et al. ${ }^{17}$ argued that SL does not require the many-body, delocalize-electron interactions of a liquid metal at all. Rather, according to them, SL is a universal property of all liquids at low enough temperatures, $T \leqq T_{c} / a$, whenever not preempted by bulk solidification. Here $T_{c}$ is the critical temperature of the liquid, and $a \approx 4-5$.

The dichotomy between these two views could be resolved, in principle, by XR measurements on selected materials ${ }^{18}$ to see which exhibit, or not, a layering peak at some $q_{\text {peak }}$. Unfortunately, practical considerations limit the number of elemental liquids that can be studied to a relatively few. One of the major problems is that the measurable $q_{z}$ range is more often than not limited to values much less than $q_{\text {peak }}$ by the strong off-specular diffuse scattering caused by thermal capillary waves. The effect of the capillary waves is to induce a surface roughness $\sigma \sim \sqrt{T / \gamma}$, where $\gamma$ is the surface tension. The consequence of this, which is shown in Fig. 1, for three liquids at room temperature, is to reduce the reflectivity $R\left(q_{z}\right)$ below that of the theoretical Fresnel reflectivity from an interface with an idealized flat, steplike surface-normal density profile. For low- $\gamma$ liquids such as water $(\sim 70 \mathrm{mN} / \mathrm{m})$ and $\mathrm{K}(\sim 100 \mathrm{mN} / \mathrm{m})$ the reduction is significant. For $\mathrm{Ga}$, where $\gamma \approx 750 \mathrm{mN} / \mathrm{m}$ the effect is almost negligible at room temperature. Even that small reduction is almost completely offset at room temperature by the SL effect that peaks at $q_{z} \approx 2.5 \AA^{-1}$. At higher temperatures, however, the effect is quite prominent. ${ }^{2}$

Although the rapid falloff in $R / R_{F}$ of water prevents its measurements out to $q_{z} \approx q_{\text {peak }}=2.0 \AA^{-1}$, our recent studies of liquid K (Ref. 19) demonstrated that if surface layering is present its signature can still be observed clearly at $q_{z}$ $\ll q_{\text {peak }}$ even for low $\gamma \lesssim 100 \mathrm{mN} / \mathrm{m}$ liquids. This is accomplished by carefully accounting for the effects of capillary waves, based on diffuse x-ray scattering (DS) measurements. We present here an $x$-ray study of the surface structure of water over the most extended $q$ range published to date, and including diffuse scattering. ${ }^{7,20}$ For the present measurements the intrinsic surface structure factor of water can be extracted directly from the raw $R\left(q_{z}\right)$ without resorting to any structural model for the interface. Comparison between the water surface structure factor, for which there is no evidence of SL, and that of $\mathrm{K}$ and Ga suggests that surface tension is not the dominant cause of the SL observed in liquid metals.

$\mathrm{X}$-ray measurements were carried out on the CMC-CAT liquid surface diffractometer, APS, Argonne National Laboratory, at a wavelength of $\lambda=1.531 \AA$. The purified water sample was contained in a Langmuir trough ${ }^{21}$ mounted on the diffractometer. The surface was periodically swept with a teflon barrier, monitoring $\gamma$ with a film balance, to ensure a clean surface. ${ }^{22}$

Both XR (Ref. 18) and DS (Ref. 23) are well-documented techniques. Experimental geometry setup employed in the experiments described in this work is shown in Fig. 2. For x-rays striking the surface at a grazing angle $\alpha$ and detected at an output angle $\beta$ in the specular plane $(\Delta \Theta=0)$, the surface-normal $(z)$ and in-plane surface-parallel (y) compo-

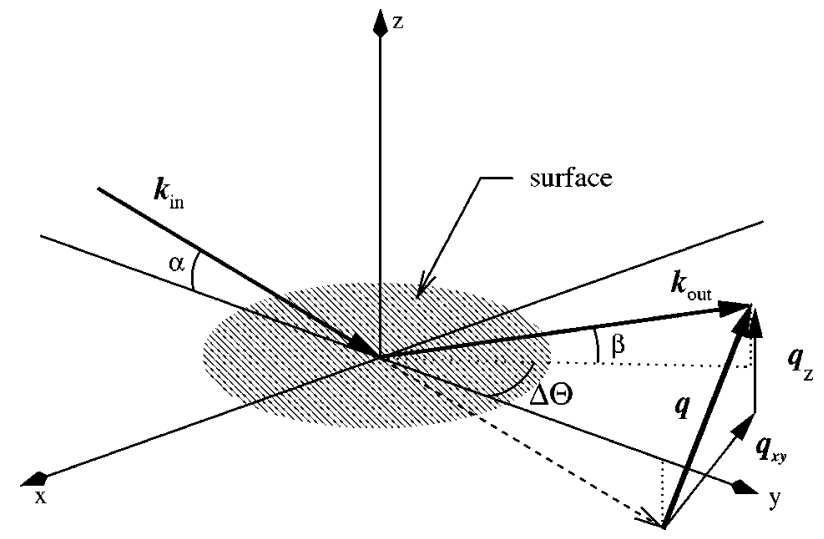

FIG. 2. Schematic description of the experimental setup.

nents of the wave vector transfer are $q_{z}=(2 \pi / \lambda)(\sin \alpha$ $+\sin \beta)$ and $q_{y}=(2 \pi / \lambda)(\cos \alpha-\cos \beta)$. In a XR measurement both angles are varied, keeping $\alpha=\beta$, so that $q_{y}=0$, and the reflected signal, measured vs $q_{z}$ and divided by the incident intensity $I_{0}$, yields $R\left(q_{z}\right)$. In a DS measurement, $\beta$ is varied for a fixed $\alpha$, and hence both $q_{z}$ and $q_{y}$ vary.

Within the Born approximation, ${ }^{18} R\left(q_{z}\right) / R_{F}\left(q_{z}\right)$ $=\left|\Phi\left(q_{z}\right)\right|^{2} W\left(\eta, q_{z}\right), \quad$ where $\Phi\left(q_{z}\right)=\left(\rho_{\infty}\right)^{-1} \int[d\langle\rho(z)\rangle /$ $d z] \exp \left(\imath q_{z} z\right) d z$ is the conventional structure factor of the liquid-vapor interface, $\langle\rho(z)\rangle$ is the intrinsic (i.e., in the absence of capillary wave smearing) surface-parallel-averaged electron-density profile along the surface-normal $z$ direction, and $W\left(\eta, q_{z}\right)$ accounts for the smearing of the intrinsic density profile by capillary waves. The aim of our XR measurement is to determine $\Phi\left(q_{z}\right)$ to observe a possible layering peak. Since both $\Phi\left(q_{z}\right)$ and $W\left(\eta, q_{z}\right)$ depend on $q_{z}$, extracting $\Phi\left(q_{z}\right)$ directly from the measured $R\left(q_{z}\right)$ is possible as $\left|\Phi\left(q_{z}\right)\right|^{2}=R\left(q_{z}\right) / W\left(\eta, q_{z}\right)$ only if $W\left(\eta, q_{z}\right)$ is known independently. For this we use the DS data.

The measured DS, shown in Fig. 3, is given by theory as $I_{D S}=\int\left[d \sigma\left(q_{x}-q_{x}^{\prime}, q_{y}-q_{y}^{\prime}, q_{z}-q_{z}^{\prime}\right) / d \Omega\right] d \omega\left(q_{x}^{\prime}, q_{y}^{\prime}, q_{z}^{\prime}\right)$, where $d \omega$ is the angular resolution of the diffractometer and

$$
\frac{d \sigma}{d \Omega}=\frac{A_{0}}{8 \pi \sin \alpha} q_{z}^{2} R_{F}\left(q_{z}\right)\left|\Phi\left(q_{z}\right)\right|^{2} \frac{\eta}{q_{x y}^{2-\eta}}\left(\frac{1}{q_{\max }}\right)^{\eta}
$$

is the scattering cross-section. Here $q_{c}$ is the critical angle for total external reflection of $\mathrm{x}$ rays, $q_{\max } \approx \pi / \xi$ is the upper cutoff for capillary wave contributions, with $\xi$ of order of the atomic diameter and $\eta=\left(k_{B} T\right) /(2 \pi \gamma) q_{z}^{2}$.

Two subtle complications are encountered in analyzing the DS data. First, nonsurface DS contributions (scattering from the bulk, sample chamber windows, etc.) can significantly distort the shape of the DS scans. These background contributions are measured by offsetting the detector by $\Delta \Theta= \pm 0.3^{\circ}$ from the plane of incidence, and are already subtracted from the data shown in Fig. 3. Second, in the fixed- $\alpha$ DS scans, carried out by scanning $\beta, q_{z}$, and thus $\left|\Phi\left(q_{z}\right)\right|^{2}$, also vary. Fortunately, $\delta q_{z} \approx \delta q_{y} / \beta$ and for the range of $q_{y}$ displayed in Fig. 3 and typical $q_{z}$ values, the changes are small enough to be neglected, e.g., a maximal 


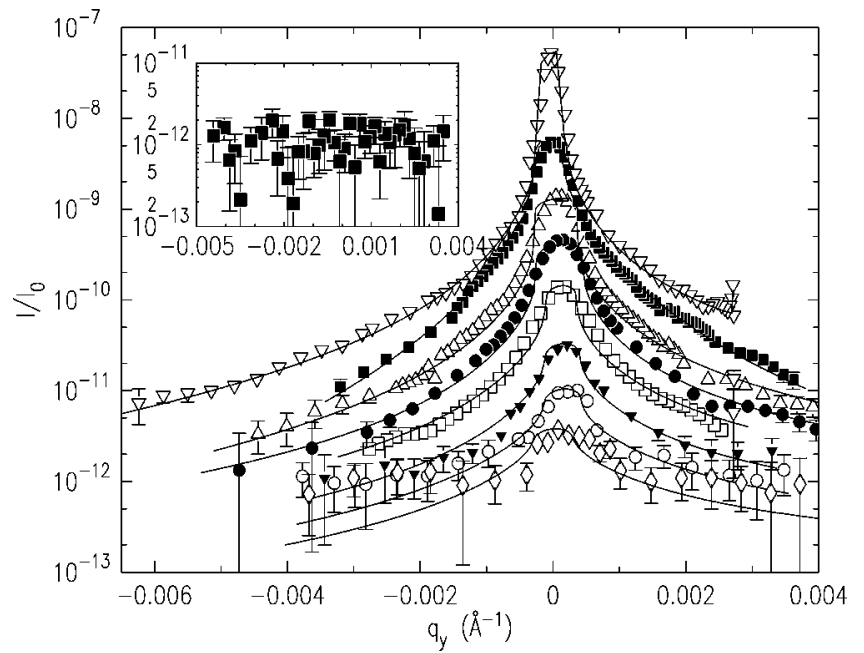

FIG. 3. Comparison of measured diffuse scattering with capillary wave theory predictions for the angles of incidence $\alpha$ (top to bottom): $2.1^{\circ}, 2.8^{\circ}, 3.5^{\circ}, 4.2^{\circ}, 5.0^{\circ}, 5.7^{\circ}, 6.0^{\circ}$, and $6.4^{\circ}$. The last data set corresponding to $7.1^{\circ}$ shown in the inset no longer exhibits a distinguishable specular peak. The $q_{z}$ values corresponding to the specular condition $q_{y}=0 \AA^{-1}$ are $0.3,0.4,0.5,0.7,0.8,0.85,0.9$, and $1.0 \AA^{-1}$, respectively.

$\delta q_{z} \approx 0.06 \AA^{-1}$ at the largest $\beta$ for $\alpha=3.5^{\circ}$. Thus, it is a good approximation for each of the DS scans to treat $\Phi\left(q_{z}\right)$ as a fixed function of $\alpha .\left|\Phi\left(q_{z}\right)\right|^{2}$ is then obtained by dividing the measured $\mathrm{DS}$ and XR curves by $W\left(\eta, q_{z}\right)$ $=\int\left[A_{0} /(8 \pi \sin \alpha)\right] q_{z}^{2} R_{F}\left(q_{z}\right)\left(\eta / q_{y}^{2-\eta}\right) q_{\max }^{-\eta} d \omega .{ }^{19}$

The theoretical curves calculated using Eq. 1 with $T$ $=298 \mathrm{~K}$ and $\gamma=72 \mathrm{mN} / \mathrm{m}$ are shown as lines in Fig. 3. As $q_{z}$ increases, so do both $\eta \propto q_{z}^{2}$ and the intensity of the offspecular power-law wings relative to that of the specular peak at $q_{y}=0$. The curve at $q_{z}=1 \AA^{-1}$ demonstrates the capillary-wave-imposed limit where the specular signal at $q_{y}=0$, which contains the surface structure information, becomes indistinguishable from the DS signal at $\pm q_{y}>0$. In principle, this limit arises from the fact that for $\eta \geqslant 2$ the singularity at $q_{y}=0$ in $d \sigma / d \Omega$ vanishes and there is no longer any criterion by which the surface scattering can be differentiated from other sources of diffuse scattering. In practice, the fact that the projection of the resolution function on the horizontal $x-y$ plane is very much wider transverse to the plane of incidence than within the plane of incidence reduces this limit to a value closer to $\eta \approx 1$. ${ }^{19}$ Figure 3 exhibits excellent agreement between the theoretical DS curves calculated from Eq. (1) with the measured DS over several decades in intensity and one decade in $\eta$, without any adjustable parameters. This confirms the applicability of the capillary wave theory for the surface of water over the $q_{z}$ range studied here, $0 \leqslant q_{z} \leqslant 0.9 \AA^{-1}$. Measurements of diffuse scattering for small $q_{z}$ (i.e., small $\eta$ ) to values of the surface parallel component of the wave vector transfer $q_{x}$ of the order of $\pi$ /atomic size have been done by Daillant et $a .^{24,25}$ by moving the detector out of the plane of incidence for grazing incident angles. At such large wave vectors the observed scattering must be interpreted as the superposition of scattering due to surface capillary waves and bulk

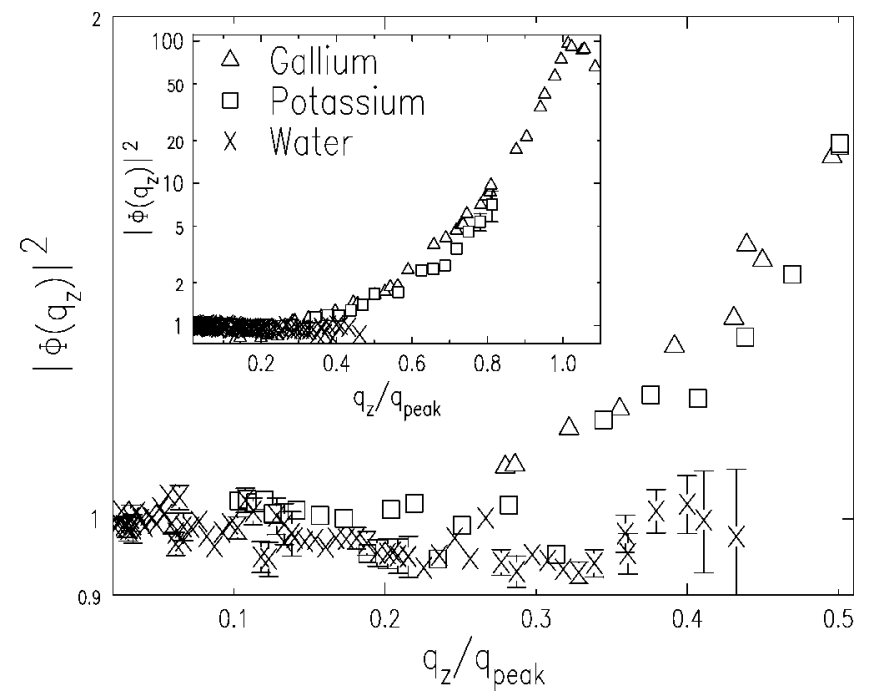

FIG. 4. Comparison of the structure factor squared $\left|\Phi\left(q_{z}\right)\right|^{2}$ for water (crosses), liquid potassium (squares), and liquid gallium (triangles). The wave vector $q_{z}$ is normalized to the expected position of the layering peak $q_{\text {peak }}$ of each sample. The inset shows the data on an extended scale. For discussion see text.

diffuse scattering from the liquid below the surface. The separation of these two contributions is rather subtle and was only accomplished through nontrivial calculations of the noncapillary terms. Eventually Daillant et al. concluded that as the value of $q_{x}$ approaches the atomic scale (i.e., $\pi /$ atomic size) the surface tension varies with $q_{x} \cdot{ }^{26}$ In principle, this dispersion should affect the sum rule that is the origin of the $1 / q_{x y}^{\eta}$ in the differential cross section in Eq. (1). On the other hand, considering that the dispersion is a relatively small effect that will only change the argument of a logarithmic term, its effect on the present analysis can be neglected.

$\left|\Phi\left(q_{z}\right)\right|^{2}$ is then obtained directly from the measured XR curve as $R\left(q_{z}\right) / W\left(\eta, q_{z}\right)$, as discussed above. It is shown in Fig. 4 (crosses) along with previously measured results for $\mathrm{K}$ (squares) and $\mathrm{Ga}$ (triangles).

The rise of the $\mathrm{Ga}\left|\Phi\left(q_{z}\right)\right|^{2}$ to $\sim 100$ at $q_{\text {peak }}$ due to layering can be clearly seen on the inset of Fig. 4. For K, the capillary-wave-imposed limit only allows obtaining $\left|\Phi\left(q_{z}\right)\right|^{2}$ for $q_{z} \lesssim 0.8 q_{\text {peak }}$. Nevertheless, the value of $\left|\Phi\left(q_{z}\right)\right|$ for $\mathrm{K}$ starts to deviate from unity for values of $q_{z} / q_{\text {peak }} \approx 0.3$. Furthermore, over the range for which it can be measured it is basically identical to the structure factor of Ga. This is a clear indication that the surface of liquid $\mathrm{K}$ has essentially the same SL as that of Ga, which is also nearly identical to that of the other liquid metals that have been studied to date, e.g., In (Ref. 27) and Sn (Ref. 28). For water, however, no deviation of $\left|\Phi\left(q_{z}\right)\right|^{2}$ from unity is observed even at the highest measurable $q_{z} / q_{\text {peak }} \approx 0.5$. This suggests that surface-induced layering does not occur at the surface of water. The different behavior, in spite of the similar $\gamma$ of water and $\mathrm{K}$, leads to the conclusion that the surface layering in $\mathrm{K}$, and by implication in other liquid metals, is not merely a consequence of its surface tension.

The absence of layering in water, and its presence in potassium, seems at first sight to corroborate the claim of Rice 
et al. ${ }^{11}$ that layering is a property arising from the metallic interaction of the liquid. On the other hand, Chacón et al.. ${ }^{17}$ who maintain that surface-induce layering is a general property of all liquids, regardless of their interactions, predict that layering should occur only at temperatures $T / T_{c} \subseteq 0.2$, where $T_{c}$ is the critical temperature of the liquid. Although supercooling is often possible, the practical limit for most reflectivity measurements is the melting temperature $T_{m}$. Thus the smallest $T / T_{c}$ for any liquid is on the order of $T_{m} / T_{c}$. For liquid metals $T_{m} / T_{c} \approx 0.15(\mathrm{~K}), 0.13(\mathrm{Hg}), 0.07(\mathrm{In}), 0.066$ $(\mathrm{Sn})$, and $0.043(\mathrm{Ga})$. Since these values are $<0.2$, by Chacón's criteria surface layering is expected, and indeed demonstrated experimentally to occur, in all of them. ${ }^{1,2,19,27,28} \mathrm{By}$ contrast, for water, where $T_{m} / T_{c}=0.42 \gg 0.2$, Chacón's criteria predict that the appearance of surface layering is preempted by bulk freezing, and thus no SL should occur at room temperature, as indeed found here.

In summary, we have shown that the surface of water does not exhibit SL even though its surface tension is not significantly different from that of liquid $\mathrm{K}$ for which SL was observed. Thus the atomic layering of liquid $\mathrm{K}$ cannot be the result of surface tension alone. Although this seems to support Rice's argument that the metallic phase is essential for SL, we note that SL is exhibited by both liquid crystals ${ }^{14}$ and other intermediate-size organic molecules. ${ }^{29}$ Consequently, there can be other criteria for SL, in addition to those proposed by Rice. One possibility, proposed by Chacón et al., is that SL should be ubiquitous for all liquids that can be cooled to temperatures of the order of $0.2 T_{c}$. Unfortunately, this is difficult to explore experimentally since suitable liquids are rather scarce. For example, liquid noble gases, the archetypi- cal van der Waals liquids, have all $T_{m} / T_{c}>0.55$. For liquid helium the ratio is lower but in view of both the presence of the superfluid transition and the low scattering cross section this is a difficult system to study. X-ray reflectivity measurements at $1 \mathrm{~K}$, i.e., $T_{m} / T_{c} \sim 0.2$, did not exhibit evidence for SL. ${ }^{9}$ Similarly, the polar liquids oxygen and fluorine have only $T_{m} / T_{c} 0.38$ and 0.37 , respectively. There are, however, some low melting organic van der Waals liquids, e.g., propane and 1-butene, that have $0.2<T_{m} / T_{c}<0.25$, and may be suitable for addressing this issue. It would be also very interesting to measure the surface structure of materials such as Se $\left(T_{m} / T_{c}=0.28\right)$, Te (0.3), Sc (0.28), and Cd (0.22), for which $T_{m} / T_{c}>0.2$. If it were possible to probe surface layering in these high $T_{m} / T_{c}$ materials such experiments could test Chacón's argument. Unfortunately, these materials have relatively high vapor pressure and that precludes the use of UHV methods for insuring atomically clean surfaces. The surfaces of alkalis ${ }^{30}$ and also mercury can be kept clean without UHV methods. For most of the experimentally accessible metals this is not true.

This work has been supported by the U.S. Department of Energy Grants Nos. DE-FG02-88-ER45379 and DEAC0298CH10886, the National Science Foundation Grant No. DMR-0124936, and the U.S.-Israel Binational Science Foundation, Jerusalem. Work at the CMC Beamlines was supported in part by the Office of Basic Energy Sciences of the U.S. Department of Energy and by the National Science Foundation Division of Materials Research. Use of the Advanced Photon Source was supported by the Office of Basic Energy Sciences of the U.S. Department of Energy under Contract No. W-31-109-Eng-38.
${ }^{1}$ O.M. Magnussen, B.M. Ocko, M.J. Regan, K. Penanen, P.S. Pershan, and M. Deutsch, Phys. Rev. Lett. 74, 4444 (1995).

${ }^{2}$ M.J. Regan, E.H. Kawamoto, S. Lee, P.S. Pershan, N. Maskil, M. Deutsch, O.M. Magnussen, B.M. Ocko, and L.E. Berman, Phys. Rev. Lett. 75, 2498 (1995).

${ }^{3}$ H. Tostmann, E. DiMasi, P.S. Pershan, B.M. Ocko, O.G. Shpyrko, and M. Deutsch, Phys. Rev. B 61, 7284 (2000); E. DiMasi, H. Tostmann, O.G. Shpyrko, P. Huber, B.M. Ocko, P.S. Pershan, M. Deutsch, and L.E. Berman, Phys. Rev. Lett. 86, 1538 (2001).

${ }^{4}$ B. Yang, D. Gidalevitz, D.X. Li, Z.Q. Huang, and S.A. Rice, Proc. Natl. Acad. Sci. U.S.A. 96, 13009 (1999); B. Yang, D.X. Li, Z.Q. Huang, and S.A. Rice, Phys. Rev. B 62, 13111 (2000).

${ }^{5}$ F.P. Buff, R.A. Lovett, and F. Stilling, Phys. Rev. Lett. 15, 621 (1965); F.H. Stillinger and J.D. Weeks, J. Phys. Chem. 99, 2807 (1995).

${ }^{6}$ J.S. Rowlinson and B. Widom, Molecular Theory of Capilarity (Oxford University Press, Oxford, 1982).

${ }^{7}$ A. Braslau, M. Deutsch, P.S. Pershan, A.H. Weiss, J. Als-Nielsen, and J. Bohr, Phys. Rev. Lett. 54, 114 (1985).

${ }^{8}$ B.M. Ocko, X.Z. Wu, E.B. Sirota, S.K. Sinha, and M. Deutsch, Phys. Rev. Lett. 72, 242 (1994).

${ }^{9}$ L.B. Lurio, T.A. Rabedeau, P.S. Pershan, I.F. Silvera, M. Deutsch, S.D. Kosowsky, and B.M. Ocko, Phys. Rev. Lett. 68, 2628 (1992); Phys. Rev. B 48, 9644 (1993).
${ }^{10}$ W.J. Huisman et al., Nature (London) 390, 107379 (1997).

${ }^{11}$ S.A. Rice, D. Guidotti, and H.L. Lemberg, W.C. Murphy, and A. N. Bloch, in Advances in Chemical Physics XXVII, edited by I. R. Prigogine and S.A. Rice (Wiley, Chichester, 1974), p. 543; M.P. D'Evelyn and S.A. Rice, J. Chem. Phys. 78, 5225 (1983); S.A. Rice, Mol. Simul. 29, 593 (2003).

${ }^{12}$ F. Celestini, F. Ercolessi, and E. Tosatti, Phys. Rev. Lett. 78, 3153 (1997); S. Iarlori, P. Carnevali, F. Ercolesi, and E. Tosatti, Surf. Sci. 211/212, 55 (1989).

${ }^{13}$ G. Fabricius, E. Artacho, D. Sanchez-Portal, P. Ordejon, D.A. Drabold, and J.M. Soler, Phys. Rev. B 60, R16 283 (1999); J.M. Soler, G. Fabricius, and E. Artacho, Surf. Sci. 482-485, 1314 (2001).

${ }^{14}$ P.S. Pershan, Phys. Today 35 (5), 34 (1982).

${ }^{15}$ K. Katsov and J.D. Weeks, J. Phys. Chem. B 106, 8429 (2002).

${ }^{16}$ A. Madsen, O. Konovalov, A. Robert, and G. Grubel, Phys. Rev. E 64, 061406 (2001).

${ }^{17}$ E. Chacon, M. Reinaldo-Falagan, E. Velasco, and P. Tarazona, Phys. Rev. Lett. 87, 166101 (2001); E. Velasco, P. Tarazona, M. Reinaldo-Falagan, and E. Chacon, J. Chem. Phys. 117, 10777 (2002).

${ }^{18}$ M. Deutsch and B.M. Ocko, in Encyclopedia of Applied Physics, edited by G.L. Trigg (VCH, New York, 1998), Vol. 23, p. 479; J. Als-Nielsen and D. McMorrow, Elements of Modern x-Ray 
Physics (Wiley, New York, 2001).

${ }^{19}$ O.G. Shpyrko, P. Huber, A. Grigoriev, P.S. Pershan, B.M. Ocko, H. Tostmann, and M. Deutsch, Phys. Rev. B 67, 115405 (2003).

${ }^{20}$ D.K. Schwartz, M.L. Schlossman, E.H. Kawamoto, G.J. Kellogg, P.S. Pershan, and B.M. Ocko, Phys. Rev. A 41, 5687 (1990).

${ }^{21}$ D.K. Schwartz, M.L. Schlossman, and P.S. Pershan, J. Chem. Phys. 96, 2356 (1992).

${ }^{22}$ G.L. Gaines, Insoluble Monolayers at Liquid-Gas Interfaces (Wiley, New York, 1966).

${ }^{23}$ P.S. Pershan, Colloids Surf., A 171, 149 (2000).

${ }^{24}$ C. Fradin, A. Braslau, D. Luzet, D. Smilgies, M. Alba, N. Boudet, K. Mecke, J. Daillant, Nature (London) 403, 871 (2000).
${ }^{25}$ S. Mora, J. Daillant, K. Mecke, D. Luzet, A. Braslau, M. Alba, and B. Struth, Phys. Rev. Lett. 90, 216101 (2003).

${ }^{26}$ K.R. Mecke and S. Dietrich, Phys. Rev. E 59, 6766 (1999).

${ }^{27}$ H. Tostmann, E. DiMasi, P.S. Pershan, B.M. Ocko, O.G. Shpyrko, and M. Deutsch, Phys. Rev. B 59, 783 (1999).

${ }^{28}$ O.G. Shpyrko, A. Grigoriev, P.S. Pershan, C. Steimer, B.M. Ocko, M. Deutsch, B. Lin, M. Meron, T. Graber, and J. Gerbhardt (unpublished).

${ }^{29}$ C.J. Yu, A.G. Richter, A. Datta, M.K. Durbin, and P. Dutta, Phys. Rev. Lett. 82, 2326 (1999).

${ }^{30}$ C.C. Addison, The Chemistry of the Liquid Alkali Metals (Wiley, New York, 1984). 